\title{
ASTRONOMICAL OBSERVATIONS OF THE PAH EMISSION BANDS
}

\author{
E. Peeters ${ }^{1,2}$
}

\begin{abstract}
The infrared (IR) spectra of many galactic and extragalactic objects are dominated by emission features at 3.3, 6.2, 7.7, 8.6 and $11.2 \mu \mathrm{m}$, generally attributed to the IR fluorescence of Polycyclic Aromatic Hydrocarbon molecules (PAHs). These PAH bands have been found in a wide variety of environments throughout the Universe and contain up to $10 \%$ of the total power output of starforming galaxies.

Ground-based telescopes, the Infrared Space Observatory (ISO) and the Spitzer Space Telescope revealed a plethora of weaker PAH bands and provided extensive evidence for significant variability in the PAH spectrum from source to source and spatially within sources. Here we review the spectral characteristics of astronomical PAHs, their dependence on the local physical conditions and the implications for the physical and chemical characteristics of the carriers.
\end{abstract}

\section{Introduction}

The IR spectra of objects associated with dust and gas - including evolved stars, reflection nebulae (RN), the interstellar medium (ISM), star-forming regions, and galaxies - are dominated by emission features at 3.3, 6.2, 7.7, 8.6 and $11.2 \mu \mathrm{m}$, the so-called unidentified infrared (UIR) bands. These bands are generally attributed to the IR fluorescence of Polycyclic Aromatic Hydrocarbon molecules (PAHs) and related species ${ }^{3}$. Observations show without any doubt that PAHs pervade the Universe. A remarkable $20-30 \%$ of the galactic IR radiation (and hence $\sim 10-15 \%$ of the total galactic power budget) is emitted in the PAH bands and $10-20 \%$ of the cosmic carbon is locked up in these molecules. Hence, it may not come as a surprise that PAHs play a crucial role in several astrophysical and astrochemical processes

\footnotetext{
${ }^{1}$ Department of Physics and Astronomy, University of Western Ontario, London, ON N6A 3K7, Canada

2 SETI Institute, 189 N. Bernardo Ave., Suite 100, Mountain View, CA 94043, USA

${ }^{3}$ In this paper, the term PAH bands/features is used to refer to these IR emission features.
} 
as, for example, heating and cooling of the ISM and grain surface chemistry. In addition, PAH intensities are used to determine the star formation rate in galaxies, one of the key indicators for understanding galaxy formation and evolution ( $c f$. Calzetti elsewhere in this volume).

Although the presence of PAHs in space is now generally accepted and PAHs are recognized as critical players in various processes, the characteristics of the emitting population remain unclear. Indeed, no single PAH molecule has been firmly identified to date. This identification is hampered by the fact that their main spectral signatures, the UIR bands, are due to vibrational modes primarily governed by the local physical conditions of the molecular environment (which is the same for all PAHs), and only show weak dependence on size and structure. However, there is no reason to despair. Although, to first order, the PAH bands are remarkably similar throughout the Universe, detailed observational studies have revealed a multitude of spectral variations in peak positions, shapes and (relative) intensities. This provides a tool to determine or further constrain the characteristics of the emitting population and to probe the physical conditions in the emitting regions. When properly calibrated, the PAH emission bands can serve as a probe of the physical conditions in regions near and far. Given their omnipresence, a PAH diagnostic toolbox will be extremely powerful.

Here, we give a concise overview of the observational properties of the PAH emission bands. Section 2 describes the astronomical PAH spectrum and Section 3 highlights the observed spectral characteristics of the PAH bands in terms of their intensity, peak position and profile. Section 4 reports on the spectral decomposition of the PAH spectra. The astronomical implications are discussed in Section 5 and Section 6 reviews the application of the PAH bands as a diagnostic tool. We conclude with a short summary and a look into the bright PAH future in Section 7.

\section{The rich PAH spectrum}

In the early 1970's, new IR instruments on ground-based and airborne telescopes revealed broad emission features at 3.3, 6.2, 7.7, 8.6 and $11.2 \mu \mathrm{m}$ (e.g. Gillett et al. 1973; Geballe et al. 1985). In the past two decades, the unprecedented views of the IR Universe offered by ground-based telescopes, the Infrared Space Observatory (ISO) and the Spitzer Space Telescope showcased the spectral wealth of the PAH spectrum (see Fig. 1). Besides the main PAH bands, a plethora of weaker bands ${ }^{4}$ may be present. In addition, deuterated PAHs, PADs, have been tentatively detected at 4.4 and $4.65 \mu \mathrm{m}$ (Peeters et al. 2004a). These bands are characteristic for the vibrational modes of PAHs (Fig. 1). In particular, the $3.3 \mu \mathrm{m}$ band is due to $\mathrm{CH}$ stretching modes and the $6.2 \mu \mathrm{m}$ band to $\mathrm{CC}$ stretching

\footnotetext{
${ }^{4}$ This can include bands at $3.4,3.5,5.25,5.75,6.0,6.6,6.9,7.2-7.4,8.2,10.5,10.8,11.0,12.0$, $13.5,14.2,15.8,16.4,16.6,17.0,17.4$ and, $17.8 \mu \mathrm{m}$. A band at $19 \mu \mathrm{m}$ was originally considered as part of the PAH spectrum. However, its spatial distribution in the reflection nebula NGC 7023 is significantly distinct from that of the PAH bands (Sellgren et al. 2007). This feature is recently firmly assigned to $\mathrm{C}_{60}$ (Cami et al. 2010; Sellgren et al. 2010, elsewhere in this volume).
} 


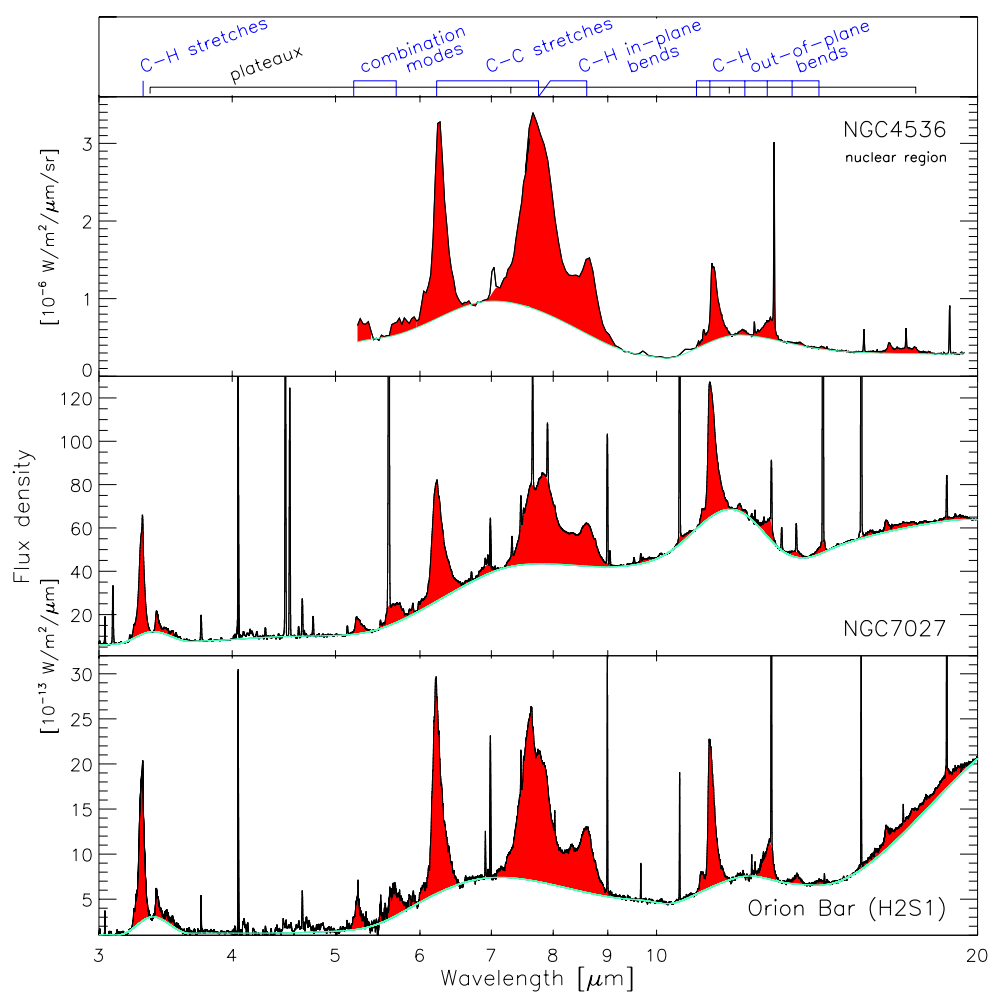

Fig. 1. The ISO-SWS spectra of the planetary nebula NGC 7027 and the PhotoDissociation Region (PDR) at the Orion Bar and, the Spitzer-IRS spectrum of the nuclear region of NGC 4536, an H II -type galaxy, (SINGS legacy program; Kennicutt et al. 2003) illustrate the richness and variety of the PAH spectrum. Also indicated are the aromatic mode identifications of the major PAH bands.

modes. $\mathrm{C}-\mathrm{H}$ in plane bending modes are responsible for the $8.6 \mu \mathrm{m}$ band, coupled $\mathrm{CC}$ stretching and $\mathrm{CH}$ in-plane bending modes for the $7.7 \mu \mathrm{m}$ complex and the $\mathrm{CH}$ out-of-plane bending modes for emission in the $10-15 \mu \mathrm{m}$ region. At wavelengths longward of $15 \mu \mathrm{m}$, the vibrations involve motions of the entire molecular skeleton and hence are more specific to each molecule. Several PAH bands exhibit highly asymmetric band profiles with a steep blue rise and a red-shaded wing (e.g., 3.4, 5.25, 6.2, $11.2 \mu \mathrm{m}$; Barker et al. 1987; Roche et al. 1996; Pech et al. 2002; Verstraete et al. 2001) while other PAH bands exhibit fairly symmetric profiles (e.g., 3.3, $8.6 \mu \mathrm{m}$ bands). The asymmetry of the $12.7 \mu \mathrm{m}$ band is exceptional as it shows a blue-shaded wing with a steep red decline. The $7.7 \mu \mathrm{m}$ complex is unique and is comprised of at least 2 components: the first peaking at $\sim 7.6 \mu \mathrm{m}$ and the second between 7.8-8 $\mu \mathrm{m}$ (e.g. Bregman 1989). Additional substructure at $8.2 \mu \mathrm{m}$ is fairly common and few sources show substructure near $7.2-7.5 \mu \mathrm{m}$ 
(e.g. Moutou et al. 1999; Peeters et al. 2002). The PAH bands are often perched on top of broad emission plateaus at roughly $3.2-3.6,6-9,11-14 \mu \mathrm{m}$ (note that various methods are applied to distinguish between PAH bands and plateaus; see Sect. 3.1 for details). Several H II regions and planetary nebulae (PNe) also have broad emission between 15-20 $\mu \mathrm{m}$ with sometimes on top the 16.4 and $17.4 \mu \mathrm{m}$ bands. This is quite different from the collection of weaker bands as seen in e.g. RNe and galaxies (e.g. Van Kerckhoven et al. 2000; Werner et al. 2004; Peeters et al. 2004b; Sellgren et al. 2007; Smith et al. 2007; Boersma et al. 2010).

These emission features are now found in almost all astronomical environments including the (diffuse) interstellar medium (ISM), the edges of molecular clouds, reflection nebulae, some $\mathrm{T}$ Tauri stars, several Herbig AeBe stars, H II regions, C-rich post-AGB stars, C-rich PNe, some C-rich WR stars, supernova remnants and, novae. In addition, they have been found throughout the Universe out to redshifts $\sim 3$ in various type of galaxies: normal galaxies, starburst galaxies, ultraluminous galaxies (ULIRGs) and AGNs. The PAH emission in galaxies is not just confined to the plane or disk of the galaxy but can also be found in their halo (e.g. Engelbracht et al. 2006; Irwin et al. 2007).

\section{Spectral variations}

To first order, the PAH bands are remarkably similar notwithstanding the large variety of environments in which they are observed. However, it is clear that the PAH bands show variations in peak positions, shapes and (relative) intensities from source to source and also spatially within extended sources. In the analysis of the $\mathrm{PAH}$ bands, various assumptions are made with respect to the characteristics of the different components contributing to the IR emission. Generally, a featureless dust continuum is assumed. However, different methods are applied with regard to the PAH bands themselves. A first method assumes the broad plateaus underlying the individual PAH features to be part of these features. In this case, both plateaus and individual features are fitted together with Lorentzians or Drude profiles (e.g. Boulanger et al. 1998; Smith et al. 2007). Alternatively, the plateaus and the features are considered to be independent and local spline continua are used to separate the components (Fig. 1, e.g. Hony et al. 2001; Peeters et al. 2002). Sometimes, the features are then fitted with Gaussian profiles. Neither approach is technically correct (see Tielens 2008 for a detailed discussion). Although these methods result in different PAH band strengths and profiles, the global trends in

spectral variations are independent of the applied method. Some key results are discussed below.

\subsection{Intensity variations}

Strong variations are observed in the relative strength of the $\mathrm{CC}$ and $\mathrm{CH}$ in-planebending modes in the 6 to $9 \mu \mathrm{m}$ range relative to the $\mathrm{CH}$ modes at 3.3 and $11.2 \mu \mathrm{m}$ (Fig. 2, e.g. Hony et al. 2001; Galliano et al. 2008). Specifically, the strength of the $3.3 \mu \mathrm{m}$ feature correlates with that of the $11.2 \mu \mathrm{m}$ feature but they 

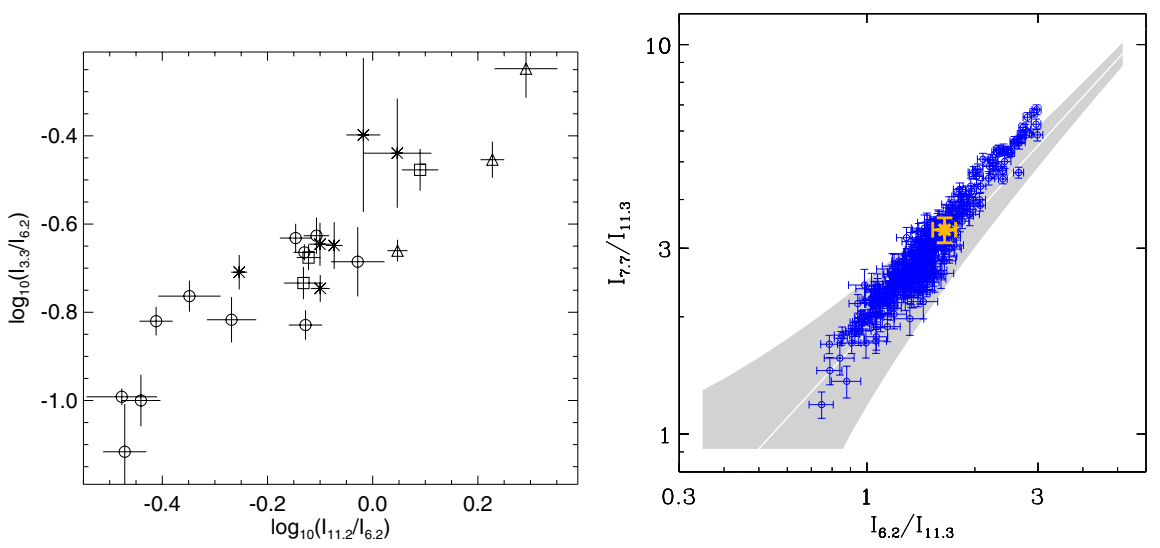

Fig. 2. Left: the correlation of the 3.3 and $11.2 \mu \mathrm{m}$ band strengths normalized to the $6.2 \mu \mathrm{m}$ band strength. Hexagons are $\mathrm{H}$ II regions, stars intermediate mass star-forming regions, squares RNe and triangles are PNe (Figure taken from Hony et al. 2001). Right: the variation in the $\mathrm{CC} / \mathrm{CH}$ band ratios within the starburst galaxy $\mathrm{M} 82$. The gray filled area represent the correlation obtained for the integrated spectra of a large sample of galactic and extragalactic sources. The white (gold) symbol is the value of the global measurement over the entire galaxy (Figure taken from Galliano et al. 2008).

both vary considerably with respect to the strengths of the $6.2,7.7$ and $8.6 \mu \mathrm{m}$ features. Likewise, the latter three PAH bands correlate well with each other. Note however that the $\mathrm{CH}$ out-of-plane (CHoop) bending modes (10-15 $\mu \mathrm{m}$ region) do not behave consistently: the $12.7 \mu \mathrm{m}$ PAH band correlates well with the 6 to $9 \mu \mathrm{m}$ modes and not with the $11.2 \mu \mathrm{m}$ PAH band. These relative intensity variations are widespread: they are seen from source to source and spatially within (extra-) galactic sources (Fig. 2). Combined, these studies reveal the interrelationship between the various PAH bands.

$\mathrm{PAH}$ ratios in Galactic and Magellanic Cloud H II regions seem to depend on metallicity (Vermeij et al. 2002; LeBouteiller et al. in prep.). A similar dependence is however not observed towards Galactic and Magellanic Cloud planetary nebulae (Bernard-Salas et al. 2009). In addition, several galaxies harboring an AGN exhibit very weak PAH emission in the 6 to $9 \mu \mathrm{m}$ region relative to the 11.2 PAH band (Smith et al. 2007; Bregman et al. 2008; Kaneda et al. 2005, elsewhere in this volume). Moreover, for these galaxies, the 7.7/11.2 PAH ratio correlates with the hardness of the radiation field. This is in contrast to galaxies with $\mathrm{H}$ II region or starburst-like characteristics where the 7.7/11.2 PAH ratio is largely insensitive to the hardness of the radiation field. Even though, the latter include low metallicity systems which typically have a harder radiation field.

The total PAH intensity is highly variable and is generally studied relative to the strength of the dust emission. The latter is commonly determined by either the dust continuum emission at the wavelength of the considered PAH band, the 
dust emission at wavelengths around 15-25 $\mu \mathrm{m}$ denoted as Very Small Grains (VSGs), the far-IR (FIR) dust emission or the total IR (TIR) dust emission. The ratio $\mathrm{PAH} / \mathrm{VSG}$ varies spatially across extended Galactic H II regions such as e.g. the Orion Bar and M 17. In particular, the dust emission dominates inside the $\mathrm{HII}$ region while in the PDR the PAHs exhibit their peak intensity and the dust continuum at 15-25 $\mu \mathrm{m}$ decreases in strength (due to its lower temperature). Similarly, the PAH/VSG ratio in galaxies depends on the hardness of radiation field and the metallicity (e.g. Madden et al. 2006; Brandl et al. 2006; Engelbracht et al. 2006; Gordon et al. 2008). These two parameters are related and so it is hard to distinguish between an origin in a less efficient PAH formation process (metallicity effect) or in an increased PAH processing (modification and/or destruction of PAHs by the hard radiation field). For a detailed discussion, we refer to Calzetti, Hunt, Galliano and Sandstrom, elsewhere in this volume.

\subsection{Profile variations}

From the early days in PAH research, it was realized that i) the $7.7 \mu \mathrm{m}$ complex is comprised of two components at 7.6 and $7.8 \mu \mathrm{m}$ which have variable relative strength (Bregman 1989; Cohen et al. 1989) and ii) the $3.3 \mu \mathrm{m}$ PAH band profile showed small variations (e.g. Tokunaga et al. 1991). However, it is the large amount of PAH spectra provided by ISO and subsequently Spitzer that allowed a systematic study of the PAH band profiles.

These PAH band profiles show pronounced variability, in particular for the CC modes (6.2 and $7.7 \mu \mathrm{m}$ bands). The variations of the band profiles for the main PAH bands are classified in three classes A, B and C (see Fig. 3; Peeters et al. 2002; van Diedenhoven et al. 2004). This classification is primarily based upon the peak position of the bands with class $\mathrm{C}$ being redshifted from class $\mathrm{B}$ which in turn is redshifted from class A. Class $\mathrm{C}$ band profiles are significantly different from class A and B. In particular, instead of a $7.7 \mu \mathrm{m}$ complex with either a dominant $7.6 \mu \mathrm{m}$ component (class A) or a dominant component peaking between 7.8 and $8 \mu \mathrm{m}$ (class B), class $\mathrm{C}$ objects show a very broad band peaking at $\sim 8.2 \mu \mathrm{m}$ with a weak to absent $8.6 \mu \mathrm{m}$ PAH band. It should also be emphasized that while class $\mathrm{A}$ and $\mathrm{C}$ show little variation in their profiles, large differences are present within class B. Some objects have band profiles that encompasses 2 classes (AB: Van Kerckhoven 2002; Boersma et al. 2008; BC: Sloan et al. 2007) and hence these three classes are not suggestive of three independent groups. Rather, the observed variations in the UIR spectra seem to span a continuous distribution going from class $\mathrm{A}$ on one extreme via class $\mathrm{B}$ to class $\mathrm{C}$ on the other extreme. The observed contrast in the magnitude of the spectral variations for the $\mathrm{CH}$ modes versus the $\mathrm{CC}$ modes is striking: the peak wavelengths of the features attributed to $\mathrm{CC}$ modes (6.2 and $7.7 \mu \mathrm{mPAH}$ bands) vary by $\sim 25$ to $50 \mathrm{~cm}^{-1}$, while the variations are smaller for the $\mathrm{CH}$ modes $(3.3,8.6$ and $11.2 \mu \mathrm{m}$ PAH bands; $\sim 4$ to $\left.11 \mathrm{~cm}^{-1}\right)$. In addition, although the classification was applied to each band individually, typically all bands in the 6-9 $\mu \mathrm{m}$ region of a single object belong 

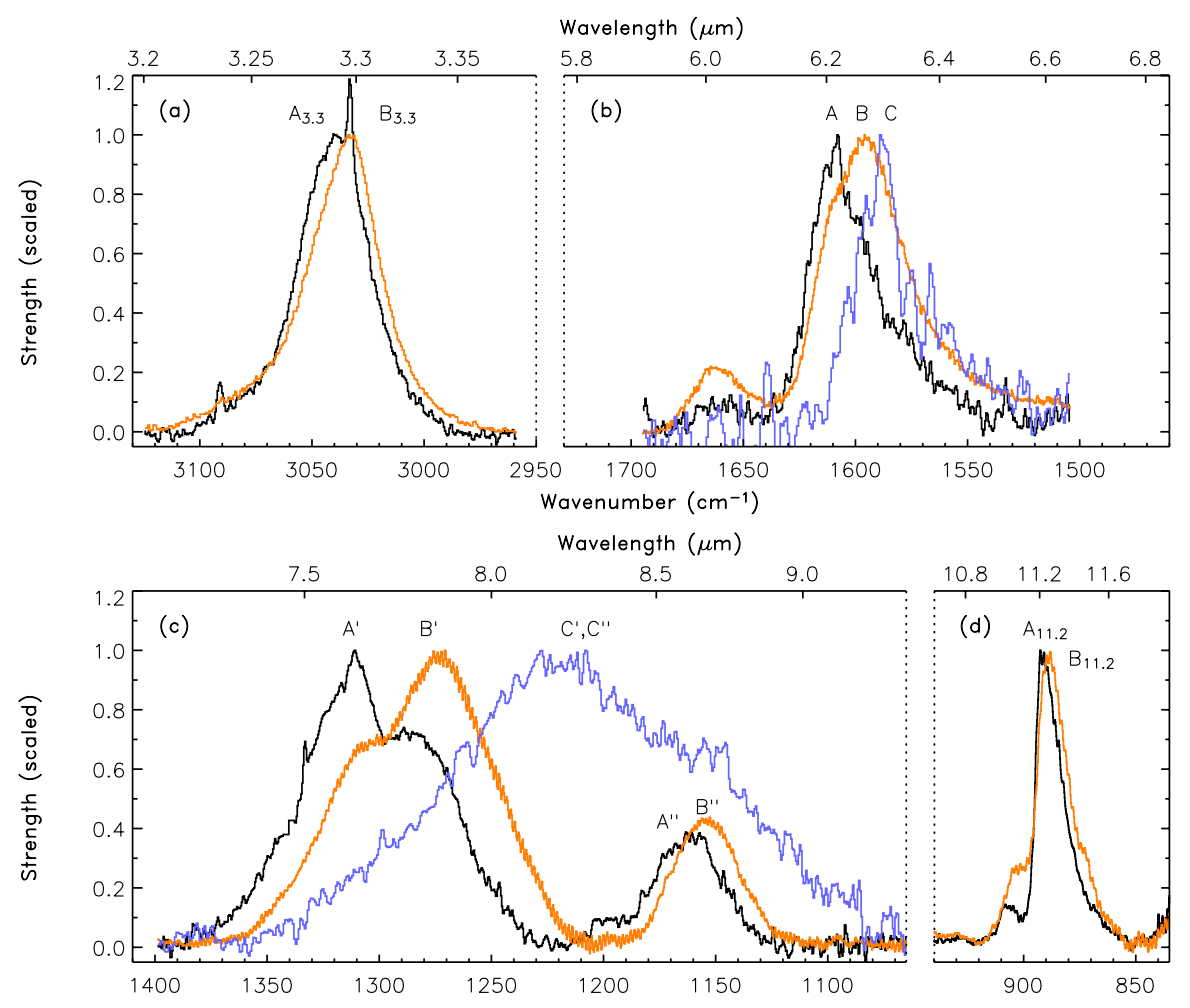

Fig. 3. The source to source variations in position and profile of the main PAH bands. In particular large variations are evident in the 6 to $9 \mu \mathrm{m}$. Class A peaks at the shortest, "nominal" wavelengths and class B at longer wavelengths. The CC modes of class C peak at even longer wavelengths (Peeters et al. 2002; Figure taken from van Diedenhoven et al. 2004).

to the same class while the $\mathrm{CH}$ modes at 3.3 and $11.2 \mu \mathrm{m}$ appear somewhat less connected to each other and/or to the PAH class in the 6-9 $\mu \mathrm{m}$ region.

As already noted by Bregman (1989) and Cohen et al. (1989) for the $7.7 \mu \mathrm{m}$ complex, the specific profile of these PAH bands depends directly on the type of object (Peeters et al. 2002; van Diedenhoven et al. 2004). Class A represents H II regions, non-isolated Herbig AeBe stars, some PNe, few post-AGB stars, RNe, the (diffuse) ISM and entire galaxies; class B contains most planetary nebulae, isolated Herbig AeBe stars and some post-AGB stars and class $\mathrm{C}$ is mainly comprised of post-AGB stars. Few HAeBe and TTauri stars are reported to have class C profiles but their PAH bands are weak and perched on top of both the dust continuum and silicate emission band. Note that post-AGB stars are found in all three classes. From a different perspective, class A profiles are associated with interstellar material (ISM) while class B and C profiles are exhibited by circumstellar material 

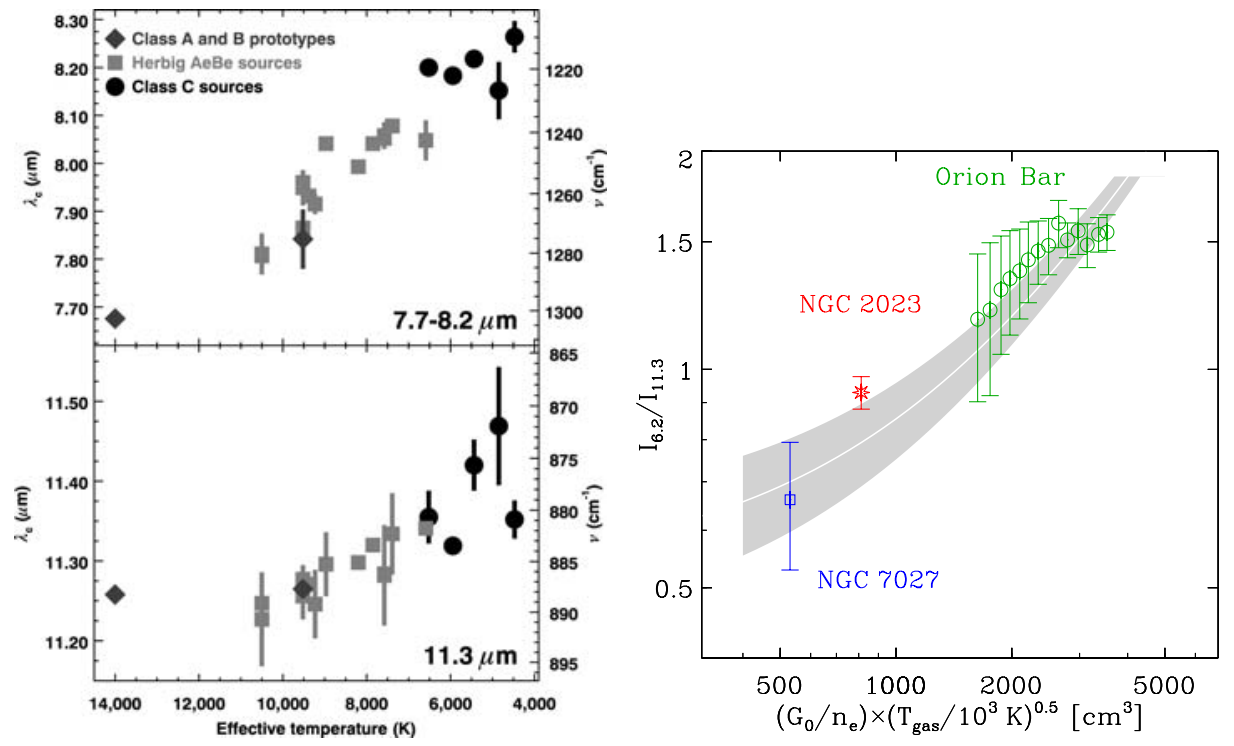

Fig. 4. Left: the central wavelengths of the 7.7-8.2 and $11.3 \mu \mathrm{m}$ PAH features plotted versus the effective temperature of the host star (Sloan et al. 2007, Figure taken from Keller et al. 2008). Right: empirical calibration of the $6.2 / 11.2$ band ratio as a function of the ionization parameter, $\mathrm{G}_{0} \mathrm{~T}^{1 / 2} / \mathrm{n}_{e}$ (Figure taken from Galliano et al. 2008).

(CSM). These spectral variations are also seen in the Magellanic Clouds (BernardSalas et al. 2009). Furthermore, they have also been observed within spatially extended objects such as RNe and evolved stars (Bregman \& Temi 2005; Song et al. 2007).

Sloan et al. (2007) found a remarkable anticorrelation of the band peak position with effective temperature of the exciting star for a sample of post-AGB stars and isolated HAeBe stars, all of class B or C (Fig. 4). This relation does not seem to hold in general because: i) PNe (belonging to class A and B) can have high effective temperatures and class B profiles (e.g. NGC 7027), ii) class A objects do not follow the correlation. For example, RNe have class A profiles and a central star with a low effective temperature and iii) the PAH bands of HAeBe stars with the same effective temperature can belong to either class A or B (Van Kerckhoven 2002; Boersma et al. 2008). Sloan et al. (2007) attributed this relation to different degrees of UV processing and argue that PAHs in reflection nebulae may have been exposed to a stronger radiation field explaining their class A PAH bands. On the other hand, the exceptions may also suggest that in addition to or in contrast with a dependence on effective temperature, other parameters such as e.g. history, environment (ISM vs. CSM), spatial structure (disk, collapsing cloud; Boersma et al. 2008) may play a role. 


\section{Spectral decomposition}

A different and potentially powerful approach to the analysis of PAH observations is the mathematical decomposition of PAH spectra as a linear combination of a basis set of components (Boissel et al. 2001; Rapacioli et al. 2005, elsewhere in this volume; Joblin et al. 2008; Berné et al. 2007, 2009, elsewhere in this volume). This method extracts spatially different components from the observations. Originally applied to RNe and edges of clouds, these studies found a basis set of three components - dubbed VSG, $\mathrm{PAH}^{0}$ and $\mathrm{PAH}^{+}$- with the VSG located deeper into the PDR while the $\mathrm{PAH}^{0}$ and $\mathrm{PAH}^{+}$are found closer to the edge of the PDR. The VSG component is characterized by broad emission bands at wavelengths slightly redshifted to those typical for PAH emission on top of a continuum and is attributed to PAH clusters. Hence, this VSG component is not the same as the VSGs discussed earlier. The components $\mathrm{PAH}^{0}$ and $\mathrm{PAH}^{+}$do not show continuum emission and are attributed to respectively neutral and ionized PAHs. This neutral PAH component does however exhibit stronger emission in the 6 to $9 \mu \mathrm{m}$ region than expected from experimental and theoretical PAH spectra. This may indicate the inclusion of some ionized PAH emission in this component. An alternative explanation may be found in the PAH size distribution. In order to fit PAH spectra of class $\mathrm{B}$ and $\mathrm{C}$, this basis set has been expanded to include templates exhibiting a very broad band at $8.2,8.3$ and $12.3 \mu \mathrm{m}$ (thus mimicking class $\mathrm{C}$ ) and a third $\mathrm{PAH}$ template, $\mathrm{PAH}^{x}$, with peak positions consistent with class $\mathrm{B}$. The latter is attributed to large ionized PAHs and is consistent with theoretical PAH spectra (see Sect. 5). These studies suggest the destruction of the VSGs or PAH clusters by UV photons resulting in the formation of PAHs. These PAH clusters may be reformed in the denser and more shielded environments of molecular clouds. In regions of high UV radiation, the $\mathrm{PAH}^{x}$ component gains in importance due to the destruction of small PAHs and the higher excitation conditions.

\section{Astronomical implications}

The interpretation of the astronomical PAH spectra strongly relies on theoretical and experimental spectroscopy of PAHs and related species. These studies reveal that intrinsic PAH spectra are determined by parameters such as the PAHs charge, size, the precise molecular (edge) structure and, temperature. Hence, a comparison of astronomical spectra with laboratory and theoretical studies allows to constrain the characteristics of the carriers and to determine the origin of the observed spectral variations. Combined with the observed behavior of PAHs in space, this gives insight into the properties of the astronomical PAH family which can be tied to specific environmental parameters. Here we name a few.

\subsection{The astronomical \& the intrinsic PAH spectra: A happy marriage}

One of the strongest criticisms against the PAH hypothesis has been the lack of a good match between a combination of experimental and/or theoretical PAH 
spectra and the astronomical PAH spectra. This is now neutralized by the good fits obtained to the three classes $\mathrm{A}, \mathrm{B}$ and $\mathrm{C}$ with the full theoretically calculated NASA Ames PAH spectral database ${ }^{5}$ (Figs. 1b and 2 of Cami elsewhere in this volume). These fits can be broken down according to size, charge and composition unveiling the underlying origin of the different classes. For example, the fit to class $\mathrm{C}$ is dominated by emission of small PAHs (number of C-atoms, $N_{C}, \leq 30$ ).

\subsection{The PAH band profiles and the composition of the PAH family}

The variations in band profiles reflect primarily a (chemical) modification of the PAH family in different environments. Currently, the precise process is not agreed upon and is further constrained by the following observations. First, the modification of the PAH family needs to be consistent with the observed PAH classes at various stages in the PAH life cycle. Second, the position of the class A $6.2 \mu \mathrm{m}$ $\mathrm{PAH}$ is not reproduced by the strongest pure CC stretching mode of pure PAHs (Peeters et al. 2002; Hudgins et al. 2005).

To resolve the second issue, various PAH-related species have been proposed. i) Hetero-atom substituted PAHs (e.g. Peeters et al. 2002; Hudgins et al. 2005; Bauschlicher et al. 2009). Substitution of a $\mathrm{C}$ atom by a $\mathrm{N}$ atom in a PAH, i.e. PANHs, systematically shifts the position of the strongest pure CC stretching mode towards shorter wavelengths while having no systematic effects on the position of other vibrational modes. This has also been noted in the decomposition of the above mentioned fits (Cami, this volume). ii) PAH-metal complexes (e.g. Hudgins et al. 2005; Bauschlicher \& Ricca 2009; Simon \& Joblin 2010; Joalland et al. 2009). These are species in which a metal atom is located either below or above the carbon skeleton. See Simon et al. (elsewhere in this volume) for specific structures. iii) PAH clusters (e.g. Rapacioli et al. 2005; Simon \& Joblin 2009). For example PAH dimers, see Rapacioli et al. (this volume) for specific structures. iv) Carbon isotope effects (Wada et al. 2003). The presence of ${ }^{13} \mathrm{C}$ in PAHs redshifts the position of the $\mathrm{CC}$ stretching modes and thus it will move the band further away from the observed astronomical class A $6.2 \mu \mathrm{m}$ PAH position. Hence, it cannot resolve the issue.

Additional mechanisms are invoked to interpret the different classes. One mechanism involves variations in the size distribution of the PAH family (Bauschlicher et al. 2008; 2009, Cami elsewhere in this volume). It was noticed that small PAHs $\left(N_{C} \leq 48\right)$ do emit at $7.6 \mu \mathrm{m}$ but do not reproduce the $7.8 \mu \mathrm{m}$ component (e.g. Peeters et al. 2002). In contrast, large PAHs $\left(54 \leq N_{C} \leq 130\right)$ emit at $7.8 \mu \mathrm{m}$ and not at $7.6 \mu \mathrm{m}$ (Bauschlicher et al. 2008, 2009). Hence, in order to reproduce the $7.7 \mu \mathrm{m}$ complex, both small and large PAHs are required and hence the variable strength of the two components (class A vs. class B) may then reflect a change of the PAH size distribution in different environments. Similarly, the broad class $\mathrm{C}$ component can be produced by small PAHs (Cami, this volume). The PAH classes may then reflect different size distributions of the PAH

\footnotetext{
${ }^{5}$ http://www.astrochem.org/pahdb/; Boersma et al. this volume.
} 
family. Alternatively, the classes represent a varying importance of aliphatics vs. aromatics (e.g. Sloan et al. 2007; Boersma et al. 2008; Keller et al. 2008; Pino et al. 2008; Acke et al. 2010). Aliphatic carbonaceous species are known to produce broader emission bands compared to PAHs and hence are put forward as the carrier of the class $\mathrm{C} 7.7 \mu \mathrm{m}$ complex. An evolution to class B and class A is then obtained by increased UV processing (e.g. due to an increase in effective temperature) of these aliphatic carbonaceous species destroying the aliphatic bonds and hence increasing the aromaticity of species. UV processing can also change the PAH size distribution and hence both mechanisms together may lay at the origin of the profile changes. While the second mechanism (towards increased aromaticity) can explain the evolutionary scenario from post-AGB stars to the ISM, it is hard to imagine a reversed process from aromatic material to more fragile aliphatic material when going from the ISM to proto-planetary environments. Hence, Boersma et al. (2008) proposed an active chemical equilibrium between aromatic and aliphatic species in all environments through hydrogenation, carbon reactions building (aliphatic) hydrocarbons and UV processing.

\subsection{The $\mathrm{CC} / \mathrm{CH}$ ratio and the charge balance of $\mathrm{PAHs}$}

Laboratory and theoretical studies on PAHs have shown the remarkable effect of ionisation on their IR spectra (e.g. Hudgins \& Allamandola 2004, Pauzat elsewhere in this volume). While peak positions are only modestly affected, the influence on intensity is striking: the bands in the $5-10 \mu \mathrm{m}$ region grow from the smallest features to become the dominant bands upon ionization. In addition, in the 10-15 $\mu \mathrm{m}$ region, the $11.0 \mu \mathrm{m}$ PAH band can be attributed to ionized PAHs while the $11.2 \mu \mathrm{m}$ band is due to neutral PAHs. Besides charge state, there are several other parameters that may influence the $\mathrm{CC} / \mathrm{CH}$ ratio (i.e. the ratio of the 6.2 or $7.7 \mu \mathrm{m}$ PAH band to the 3.3 or $11.2 \mu \mathrm{m}$ PAH band) including dehydrogenation, a modification of the temperature distribution (due to a change in the size distribution or the radiation field) and extinction. However, several studies indicate that the observed variation in the $\mathrm{CC} / \mathrm{CH}$ ratio is dominated by a variation in the degree of ionization of the PAHs in these different environments (Joblin et al. 1996; Hony et al. 2001; Galliano et al. 2008 and Galliano elsewhere in this volume).

\subsection{Molecular edge structure of PAHs}

The peak wavelength of the CHoop bending modes depends strongly on the number of adjacent peripheral C-atoms bonded to an H-atom (Bellamy 1958; Hony et al. 2001). While the exact peak position depends slightly on the charge state, the 11.0 and 11.2 $\mathrm{PAH}$ bands originate in solo $\mathrm{CH}$ groups (i.e. no H-atom is attached to adjacent $\mathrm{C}$-atoms). Both duo and trio $\mathrm{CH}$ groups (respectively two and three adjacent $\mathrm{C}$-atoms each with an $\mathrm{H}$-atom attached) contribute to the $12.7 \mu \mathrm{m}$ band, trio $\mathrm{CH}$ groups produce the $13.5 \mu \mathrm{m}$ band and quatro $\mathrm{CH}$ groups the $14.2 \mu \mathrm{m}$ band. The observed relative intensities of the CHoop bands can then be combined with the intrinsic strengths of these modes to determine possible PAH structures 
(Hony et al. 2001; Bauschlicher et al. 2008, 2009). After all, solo-CH groups decorate the long, smooth straight edges while the other $\mathrm{CH}$ groups are characteristics for corners and bays in the (irregular) edge structure. PAHs associated with PNe are characterized by very compact molecular structures with long smooth PAH edges while interstellar PAHs have more corners, either because they are on average smaller or they are more irregular larger species. Similarly, the CH stretching mode of $\mathrm{H}$ atoms located in bay regions emit around $\sim 3.22 \mu \mathrm{m}$, a region where little emission is found in astronomical PAH spectra. Therefore, the presence of bay regions in the edge structure of (small) PAHs is very low (Bauschlicher et al. 2009).

\section{PAHs as a diagnostic tool}

The $\mathrm{CC} / \mathrm{CH}$ ratio is determined by the charge balance of the PAHs. The PAH charge is set by the ratio of the ionization rate to the recombination rate, that is proportional to $\mathrm{G}_{0} \mathrm{~T}^{1 / 2} / \mathrm{n}_{e}$ where $\mathrm{G}_{0}$ is the $\mathrm{UV}$ radiation field, $\mathrm{T}$ the gas temperature and $\mathrm{n}_{e}$ the electron density (Tielens 2008). Thus, by coupling the variations in the PAH bands with known variations in the physical conditions (as derived by for example PDR models), an empirical calibration can be established that relates the PAH bands to the local physical conditions. Such a calibration will allow the determination of the physical conditions based upon the omnipresent PAH bands. This can then serve as a diagnostic tool for regions where, for example, the main PDR coolants are not easily observable, such as galaxies at large distances. This approach has been tested for a sample of three well studied objects and has been proven very promising (Fig. 4, Galliano et al. 2008; Galliano in this volume). Similarly, combining the observed $\mathrm{PAH}$ ionization ratio and $\mathrm{H}_{2}$ line ratios in dense, highly irradiated PDRs allows to derive the physical conditions (Berné et al. 2009, elsewhere in this volume).

Since PAHs are excited by UV radiation, $\mathrm{PAH}$ features are also particularly bright in massive star-forming regions. Combined with their omnipresence, this make PAHs a powerful tracer of star formation throughout the Universe (Calzetti and Hunt et al. in this volume and references therein). They are widely used to derive star formation rates of galaxies. And in combination with emission lines, they serve as diagnostics for the ultimate physical processes powering galactic nuclei (e.g. Genzel et al. 1998; Lutz et al. 1998; Peeters et al. 2004c; Sajina et al. 2007; Smith et al. 2007). Finally, the presence of PAHs is used to distinguish between shocked gas and PDRs (van den Ancker et al. 2000) and to determine redshifts in distant galaxies (e.g. Yan et al. 2007).

\section{Future}

This paper reviews the large progress made over the past 25 years in understanding the unidentified infrared bands. Nevertheless, several key questions remain regarding astronomical PAHs including: How do the PAH characteristics (e.g. 
size, charge state) interact with and reflect the physical conditions of their environment (e.g. density, radiation field, temperature, metallicity)?; Do we observe long wavelength counterparts to the well-known mid-IR PAH bands? What are their characteristics?; How can we use the UIR bands as a probe of the physical conditions in regions near and far?; Which specific molecules make up the astronomical PAH family?

The observational future for PAH research is very bright. Currently, we are fortunate to obtain the necessary observations with the Herschel Space Observatory to explore the far-IR modes of the PAHs and to couple the PAH band characteristics to the physical conditions of the environment ( $c f$. Joblin et al., elsewhere in this volume). In addition, in the near future, we will use SOFIA and the James Webb Space Telescope (JWST). But the wealth of PAH emission bands and the large variability of the PAH spectrum already prompt more questions than can be answered with the current laboratory and theoretical data. If we intend to make significant progress in our understanding of the PAH bands, the observational effort needs to be balanced with dedicated laboratory and theoretical studies. Indeed, we can only fully exploit the treasure of information hidden in the PAH emission bands by a joint effort of the observational, experimental and theoretical tools.

\section{References}

Acke, B., Bouwman, J., Juhász, A., et al., 2010, ApJ, 718, 558

Barker, J.R., Allamandola, L.J., \& Tielens, A.G.G.M., 1987, ApJ, 315, L61

Bauschlicher, C.W.Jr, Peeters, E., \& Allamandola, L.J., 2008, ApJ, 678, 316

Bauschlicher, C.W.Jr, Peeters, E., \& Allamandola, L.J., 2009, ApJ, 697, 311

Bauschlicher, C.W.Jr, \& Ricca, A., 2009, ApJ, 698, 275

Bellamy, L., 1958, The infra-red spectra of complex molecules, 2nd ed. (New York: John Wiley \& Sons, Inc.)

Bernard-Salas, J., Peeters, E., Sloan, G.C., et al., 2009, ApJ, 699, 1541

Berné, O., Joblin, C., Deville, Y., et al., 2007, A\&A, 469, 575

Berné, O., Fuente, A., Goicoechea, J.R., et al., 2009, A\&A, 706, L160

Boersma, C., Bouwman, J., Lahuis, F., et al., 2008, A\&A, 484, 241

Boersma, C., Bauschlicher, C.W.Jr, Allamandola, L.J., et al., 2010, A\&A, 511, 32

Boissel, P., Joblin, C., \& Pernot, P., 2001, A\&A, 373, L5

Boulanger, F., Boisssel, P., Cesarsky, D., \& Ryter, C., 1998, A\&A, 339, 194

Brandl, B.R., Bernard-Salas, J., Spoon, H.W.W., et al., 2006, ApJ, 653, 1129

Bregman, J. 1989, in IAU Symp. 135: Interstellar Dust, ed. Allamandola L.J., \& Tielens, A.G.G.M., 109

Bregman, J., \& Temi, P., 2005, A\&A, 621, 831

Bregman, J.D., Bregman, J.N., \& Temi, P., 2008, ASPC, 381, 34

Cami, J., Bernard-Salas, J., Peeters, E., \& Malek, S.E., 2010, Science, 329, 1180

Cohen, M., Tielens, A.G.G.M., \& Bregman, J., et al., 1989, ApJ, 341, 246

Engelbracht, C.W., Kundurthy, K.D., Gordon, K.D., et al., 2006, ApJ, 642, L127 
Galliano, F., Madden, S., Tielens, A.G.G.M., et al., 2008, ApJ, 679, 310

Geballe, T.R., Lacy, J.H., Persson, S.E., McGregor, P.J., \& Soifer, B.T., 1985, ApJ, 292, 500

Genzel, R., Lutz, D., Sturm, E., et al., 1998, ApJ, 498, 579

Gillett, F.C., Forrest, W.J., \& Merril, K.M., 1973, ApJ, 183, 87

Gordon, K.D., Engelbracht, C.W., Rieke, G.H., et al., 2008, ApJ, 682, 336

Hony, S., Van Kerckhoven, C., Peeters, E., et al., 2001, A\&A, 370, 1030

Hudgins, D.M., Bauschlicher, C.W. Jr., \& Allamandola, L.J., 2005, ApJ, 632, 316

Hudgins, D.M., \& Allamandola, L.J., 2004, Astrophys. Dust, ASP Conf. Ser., 309, 665

Irwin, J.A., Kennedy, H., Parkin, T., \& Madden, S., 2007, A\&A, 474, 461

Joalland, B., Simon, A., Marsden, C.J., \& Joblin, C., 2009, A\&A, 494, 969

Joblin, C., Szczerba, R., Berné, O., \& Szyszka, C., 2008, A\&A, 490, 189

Joblin, C., Tielens, A.G.G.M., Geballe, T.R., \& Wooden, D.H., 1996, ApJ, 460, L119

Kaneda, H., Onaka, T., \& Sakon, I., 2005, ApJ, 632, L83

Keller, L.D., Sloan, G.C., Forrest, W.J., et al., 2008, ApJ, 684, 411

Kennicutt, R.C.Jr., Armus, L., Bendo, G., et al., 2003, PASP, 115, 928

Lutz, D., Spoon, H.W.W., Rigopoulou, D., Moorwood, A.F.M., \& Genzel, R., 1998, ApJ, 505, L103

Madden, S., Galliano, F., Jones, A.P., \& Sauvage, M., 2006, A\&A, 446, 877

Moutou, C., Sellgren, K., Verstraete, L., \& Léger, A., 1999, A\&A, 347, 949

Pech, C., Joblin, C., \& Boissel, P., 2002, A\&A, 388, 639

Peeters, E., Hony, S., van Kerckhoven, C., et al., 2002, A\&A, 390, 1089

Peeters, E., Allamandola, L.J., Bauschlicher, C.W.Jr, et al., 2004a, ApJ, 604, 252

Peeters, E., Mattioda, A.L., Hudgins, D.M., \& Allamandola L.J., 2004b, ApJ, 617, L65

Peeters, E., Spoon, H.W.W., \& Tielens, A.G.G.M., 2004c, ApJ, 613, 986

Pino, T., Dartois, E., Cao, A.-T., et al., 2008, A\&A 490, 665

Rapacioli, M., Joblin, C., \& Boissel, P., 2005, A\&A, 429, 193

Roche, P.F., Lucas, P.W., \& Geballe, T.R., 1996, MNRAS, 281, L25

Sajina, A., Yan, L., \& Armus, L., et al., 2007, ApJ, 664, 713

Sellgren, K., Uchida, K.I., \& Werner, M.W., 2007, ApJ, 659, 1338

Sellgren, K., Werner, M.W., \& Ingals, J., et al., 2010, ApJ, 722, L54

Simon, A., \& Joblin, C., 2009, J. Phys. Chem. A, 113, 4878

Simon, A., \& Joblin, C., 2010, ApJ, 712, 69

Sloan, G.C., Jura, M., Duley, W.W., et al., 2007, ApJ, 664, 1144

Smith, J.D., Draine, B.T., Dale, D.A., et al., 2007, ApJ, 656, 770

Song, I.-O., McCombie, J., Kerr, T.H., \& Sarre, P.J., 2007, MNRAS, 380, 979

Tielens, A.G.G.M., 2008, ARA\&A, 46, 289

Tokunaga, A.T., Sellgren, K., Smith, R.G., et al., 1991, ApJ, 380, 452

van den Ancker, M.E., Tielens, A.G.G.M., \& Wesselius, P.R., 2000, A\&A, 358, 1035

van Diedenhoven, B., Peeters, E., Van Kerckhoven, C., et al., 2004, ApJ, 611, 928

Van Kerckhoven, C., 2002, Ph.D. Thesis, Catholic University of Leuven

Van Kerckhoven, C., Hony, S., Peeters, E., et al., 2000, A\&A, 357, 1013 
Vermeij, R., Peeters, E., Tielens, A.G.G.M., \& van der Hulst, J.M., 2002, A\&A, 382, 1042

Verstraete, L., Pech, C., Moutou, C., et al., 2001, A\&A, 372, 981

Wada, S., Onaka, T., Yamamura, I., Murata, Y., \& Tokunaga, A.T., 2003, A\&A, 407, 551

Werner, M.W., Uchida, K.I., Sellgren, K., et al., 2004, ApJS, 154, 309

Yan, L., Sajina, A., Fadda, D., et al., 2007, ApJ, 658, 778 